\title{
Studies on stress- strain behaviour of fibre reinforced self- compacting concrete in confined state
}

\author{
Gorla Jayasri ${ }^{1 *}, V$ Siva Prasad Raju ${ }^{2}$, Srinivasa Reddy $\mathrm{V}^{3}$ and $M$ Mounika $^{4}$ \\ ${ }^{1}$ M. Tech (Structural Engineering), Department of Civil Engineering, GRIET, Hyderabad, India. ${ }^{1}$ \\ ${ }^{2}$ Assistant Professor of Civil Engineering, GRIET, Hyderabad, India. \\ ${ }^{3}$ Professor of Civil Engineering, GRIET, Hyderabad, India. \\ ${ }^{4}$ Assistant Professor of Civil Engineering, VJIT, Hyderabad, India.
}

\begin{abstract}
In the present study, the stress-stain behaviour of self-compacting concrete (SCC) and fibre reinforced self-compacting concrete (FRSCC) were taken up. The stress-strain behaviour was studied for the SCC and FRSCC mixes in unconfined and confined states. The confinement was given in the form of steel hoops in the cylinders, 3 hoops $(0.8 \%)$, 4 hoops $(1.1 \%), 5$ hoops $(1.3 \%)$ and 6 hoops $(1.6 \%)$. The addition of fibres along with confinement of FRSCC with steel hoops enhanced the compressive strength, indicating further confinement effect in the FRSCC. It is observed that the addition of fibres is helpful in lower confinements only. Beyond $1.1 \%$ confinement, the addition of any type of fibres doesn't show any effect on compressive strengths. From the stress-strain behaviour of all types of FRSCC, it is concluded that the ultimate load-carrying capacity and strains at peak stresses are more in SFRSCC and HFRSCC for mixes up to $1.1 \%$ confinement. The addition of fibres to SCC has increased the ductility in both confined and unconfined states
\end{abstract}

\section{Introduction}

Self-Compacting concrete, originally developed in Japan has given answers to many mechanical and durability problems and enhanced the strength and durability characteristics of concrete. Introduction of fibres in SCC has further improved its characteristics like crack, resistance, plasticity, impact resistance, durability etc., The Studies on Stress-Strain behaviour of concrete are essential in determining the parameters like energy absorption, toughness, plasticity index and they are very useful in design of structures using such concretes. Further modelling the stress-strain behaviour helps in predicating their behaviour.

\section{Methodology}

In this phase of investigations, the stress-stain behaviour of self-compacting concrete (SCC) and fibre reinforced self-compacting concrete (FRSCC) were taken up. The stress-strain behaviour was studied for the SCC and FRSCC mixes in unconfined and confined states. The confinement was given in the form of steel hoops in the cylinders, 3 hoops $(0.8 \%), 4$ hoops $(1.1 \%), 5$ hoops $(1.3 \%)$ and 6 hoops $(1.6 \%)$ as shown in Fig 1 . The tests were carried out on the standard cylindrical specimens of diameter $150 \mathrm{~mm}$ and height $300 \mathrm{~mm}$. After casting, the cylinders were capped with cement mortar and cured for a period of 28 days in curing tanks. The specimens were then taken out and made surface dry. The samples were placed in a microprocessor strain controlled universal testing machine of $1000 \mathrm{kN}$ capacity and tested under uni-axial compression as per IS 516:1959. The stressstrain behaviour as obtained was plotted.

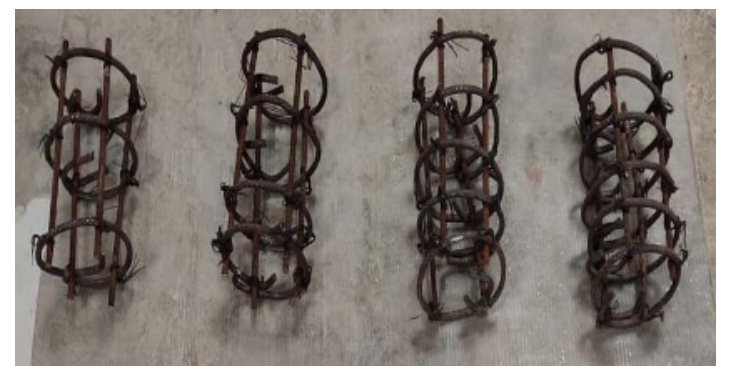

Fig.1. Cylinders with different Confinements

${ }^{*}$ Corresponding author: jayasrigorla2510@gmail.com 
Table 1. Percentage of confinement by volume

\begin{tabular}{|c|c|c|}
\hline & & Volume of confinement percentage \\
\hline C0 & Confinement 0 hoops & 0.00 \\
\hline C3 & Confinement 3 hoops & 0.80 \\
\hline C4 & Confinement 4 hoops & 1.06 \\
\hline C5 & Confinement 5 hoops & 1.33 \\
\hline C6 & Confinement 6 hoops & 1.59 \\
\hline
\end{tabular}

Table 2. Stress-strain values of plain self-compacting concrete (PSCC) mixes made with $\mathrm{PF}=1.12$ and $\mathrm{s} / \mathrm{a}=0.53$ in confined state

\begin{tabular}{|c|c|c|c|c|c|c|c|c|c|}
\hline \multicolumn{10}{|c|}{$\mathrm{PF}=1.12$ and $\mathrm{s} / \mathrm{a}=0.53$} \\
\hline \multicolumn{2}{|c|}{ M30PSCC-C0 } & \multicolumn{2}{|c|}{ M30PSCC-C3 } & \multicolumn{2}{|c|}{ M30PSCC-C4 } & \multicolumn{2}{|c|}{ M30PSCC-C5 } & \multicolumn{2}{|c|}{ M30PSCC-C6 } \\
\hline Strain & Stress & Strain & Stress & Strain & Stress & Strain & Stress & Strain & Stress \\
\hline 0.0000 & 0.00 & 0.0000 & 0.00 & 0.0000 & 0.00 & 0.0000 & 0.00 & 0.0000 & 0.00 \\
\hline 0.0002 & 3.01 & 0.0001 & 2.76 & 0.0060 & 0.00 & 0.0000 & 0.22 & 0.0000 & 0.26 \\
\hline 0.0003 & 6.18 & 0.0003 & 5.91 & 0.0000 & 45.20 & 0.0003 & 5.86 & 0.0003 & 5.22 \\
\hline 0.0005 & 9.86 & 0.0006 & 10.05 & 0.0000 & 0.39 & 0.0005 & 10.19 & 0.0006 & 10.96 \\
\hline 0.0007 & 13.37 & 0.0008 & 13.40 & 0.0004 & 7.83 & 0.0008 & 14.96 & 0.0009 & 15.91 \\
\hline 0.0010 & 17.55 & 0.0011 & 16.95 & 0.0007 & 12.52 & 0.0010 & 18.21 & 0.0012 & 20.87 \\
\hline 0.0013 & 22.39 & 0.0012 & 20.30 & 0.0010 & 16.04 & 0.0012 & 23.42 & 0.0015 & 25.30 \\
\hline 0.0015 & 25.57 & 0.0015 & 23.85 & 0.0012 & 20.35 & 0.0015 & 26.44 & 0.0018 & 29.22 \\
\hline 0.0018 & 29.08 & 0.0016 & 26.60 & 0.0014 & 25.43 & 0.0018 & 30.78 & 0.0021 & 32.87 \\
\hline 0.0019 & 32.42 & 0.0019 & 29.37 & 0.0018 & 28.17 & 0.0022 & 34.45 & 0.0024 & 36.00 \\
\hline 0.0022 & 34.27 & 0.0021 & 32.32 & 0.0020 & 31.70 & 0.0026 & 37.91 & 0.0026 & 39.13 \\
\hline 0.0026 & 38.31 & 0.0024 & 34.69 & 0.0024 & 35.02 & 0.0029 & 39.20 & 0.0029 & 42.78 \\
\hline 0.0027 & 34.96 & 0.0025 & 36.86 & 0.0027 & 37.57 & 0.0030 & 40.07 & 0.0032 & 45.91 \\
\hline 0.0030 & 33.80 & 0.0028 & 37.46 & 0.0031 & 40.30 & 0.0033 & 41.79 & 0.0037 & 49.04 \\
\hline 0.0032 & 30.48 & 0.003 & 42.48 & 0.0033 & 44.91 & 0.0037 & 47.06 & 0.0040 & 50.61 \\
\hline 0.0035 & 27.50 & 0.0031 & 38.86 & 0.0035 & 39.13 & 0.0038 & 41.54 & 0.0044 & 56.76 \\
\hline 0.0037 & 23.67 & 0.0034 & 37.88 & 0.0037 & 35.80 & 0.0041 & 40.01 & 0.0045 & 50.61 \\
\hline 0.0039 & 21.01 & 0.0037 & 35.34 & 0.0039 & 33.46 & 0.0043 & 37.61 & 0.0048 & 48.26 \\
\hline 0.0042 & 18.53 & 0.0039 & 33.59 & 0.0041 & 31.30 & 0.0045 & 36.07 & 0.0050 & 47.48 \\
\hline 0.0044 & 16.03 & 0.0042 & 31.83 & 0.0043 & 28.96 & 0.0047 & 34.33 & 0.0051 & 45.13 \\
\hline 0.0046 & 15.54 & 0.0044 & 29.09 & 0.0046 & 26.61 & 0.0050 & 31.70 & 0.0052 & 42.00 \\
\hline & & 0.0045 & 26.74 & & & & & 0.0054 & 39.65 \\
\hline & & 0.0047 & 24.98 & & & & & & \\
\hline & & 0.0049 & 23.42 & & & & & & \\
\hline
\end{tabular}

Table 3. Stress-strain values of plain self-compacting concrete (PSCC) mixes made with $\mathrm{PF}=1.14$ and $\mathrm{s} / \mathrm{a}=0.57$ in confined state

\begin{tabular}{|c|c|c|c|c|c|c|c|c|c|}
\hline \multicolumn{10}{|c|}{$\mathrm{PF}=1.14$ and $\mathrm{s} / \mathrm{a}=0.57$} \\
\hline \multicolumn{2}{|c|}{ M30PSCC-C0 } & \multicolumn{2}{|c|}{ M30PSCC-C3 } & \multicolumn{2}{|c|}{ M30PSCC-C4 } & \multicolumn{2}{|c|}{ M30PSCC-C5 } & \multicolumn{2}{|c|}{ M30PSCC-C6 } \\
\hline Strain & Stress & Strain & Stress & Strain & Stress & Strain & Stress & Strain & Stress \\
\hline 0.0000 & 0.00 & 0.0000 & 0.00 & 0.0000 & 0.00 & 0.0000 & 0.00 & 0.0000 & 0.00 \\
\hline 0.0000 & 0.17 & 0.0002 & 4.50 & 0.0000 & 0.66 & 0.0000 & 0.26 & 0.0005 & 8.61 \\
\hline 0.0000 & 0.34 & 0.0005 & 8.36 & 0.0002 & 4.80 & 0.0003 & 5.93 & 0.0007 & 14.09 \\
\hline 0.0002 & 4.48 & 0.0008 & 13.93 & 0.0004 & 8.95 & 0.0005 & 11.35 & 0.0010 & 19.57 \\
\hline 0.0005 & 8.97 & 0.0012 & 18.43 & 0.0008 & 12.66 & 0.0007 & 15.22 & 0.0012 & 23.22 \\
\hline 0.0008 & 12.59 & 0.0014 & 22.29 & 0.0011 & 18.56 & 0.0009 & 19.08 & 0.0016 & 30.26 \\
\hline 0.0011 & 16.72 & 0.0017 & 26.79 & 0.0015 & 22.93 & 0.0011 & 23.73 & 0.0019 & 34.96 \\
\hline 0.0013 & 20.52 & 0.0020 & 30.21 & 0.0017 & 26.42 & 0.0014 & 26.81 & 0.0022 & 38.61 \\
\hline 0.0017 & 23.62 & 0.0022 & 33.86 & 0.0020 & 31.00 & 0.0017 & 31.45 & 0.0026 & 42.52 \\
\hline 0.0020 & 27.24 & 0.0025 & 36.43 & 0.0023 & 34.72 & 0.0021 & 36.08 & 0.0030 & 45.39 \\
\hline 0.0023 & 29.48 & 0.0027 & 37.93 & 0.0026 & 37.55 & 0.0024 & 38.91 & 0.0033 & 48.00 \\
\hline 0.0027 & 34.48 & 0.0029 & 40.07 & 0.0029 & 39.30 & 0.0027 & 42.51 & 0.0035 & 49.30 \\
\hline
\end{tabular}




\begin{tabular}{|l|l|l|l|l|l|l|l|l|l|}
\hline 0.0029 & 36.90 & 0.0030 & 42.21 & 0.0033 & 44.54 & 0.0031 & 45.33 & 0.0037 & 51.13 \\
\hline 0.0033 & 36.90 & 0.0033 & 42.00 & 0.0036 & 44.76 & 0.0033 & 45.32 & 0.0041 & 52.17 \\
\hline 0.0035 & 34.66 & 0.0036 & 41.14 & 0.0039 & 43.01 & 0.0035 & 46.60 & 0.0043 & 50.61 \\
\hline 0.0037 & 31.38 & 0.0039 & 38.79 & 0.0041 & 40.39 & 0.0036 & 47.37 & 0.0044 & 48.78 \\
\hline 0.0040 & 28.28 & 0.0041 & 36.00 & 0.0043 & 37.55 & 0.0040 & 44.50 & 0.0047 & 45.13 \\
\hline 0.0042 & 25.86 & 0.0042 & 33.00 & 0.0045 & 35.59 & 0.0044 & 41.37 & 0.0049 & 41.74 \\
\hline 0.0043 & 24.14 & 0.0044 & 30.86 & 0.0046 & 34.06 & 0.0047 & 39.29 & 0.0052 & 37.57 \\
\hline 0.0045 & 22.07 & 0.0045 & 28.29 & & & 0.0051 & 37.45 & & \\
\hline 0.0047 & 20.86 & & & & & & & & \\
\hline
\end{tabular}

Table 4. Stress-strain values of glass fibre reinforced self-compacting concrete (GFRSCC) mixes made with $P F=1.12$ and s/a=0.53 in confined state

\begin{tabular}{|c|c|c|c|c|c|c|c|c|c|}
\hline \multicolumn{10}{|c|}{$\mathrm{PF}=1.12$ and $\mathrm{s} / \mathrm{a}=0.53$} \\
\hline \multicolumn{2}{|c|}{ M30GFRSCC-C0 } & \multicolumn{2}{|c|}{ M30GFRSCC-C3 } & \multicolumn{2}{|c|}{ M30GFRSCC-C4 } & \multicolumn{2}{|c|}{ M30GFRSCC-C5 } & \multicolumn{2}{|c|}{ M30GFRSCC-C6 } \\
\hline Strain & Stress & Strain & Stress & Strain & Stress & Strain & Stress & Strain & Stress \\
\hline 0.0000 & 0.00 & 0.0000 & 0.00 & 0.0000 & 0.00 & 0.0000 & 0.00 & 0.0000 & 0.00 \\
\hline 0.0004 & 6.00 & 0.0001 & 2.36 & 0.0000 & 0.19 & 0.0000 & 0.00 & 0.0000 & 0.52 \\
\hline 0.0006 & 10.00 & 0.0003 & 5.70 & 0.0001 & 2.33 & 0.0003 & 4.36 & 0.0003 & 6.01 \\
\hline 0.0009 & 14.00 & 0.0006 & 10.81 & 0.0003 & 5.04 & 0.0005 & 10.46 & 0.0005 & 9.93 \\
\hline 0.0012 & 17.00 & 0.0009 & 14.54 & 0.0006 & 8.73 & 0.0009 & 14.17 & 0.0008 & 16.46 \\
\hline 0.0015 & 22.00 & 0.0011 & 17.49 & 0.0009 & 12.22 & 0.0013 & 20.07 & 0.0011 & 21.16 \\
\hline 0.0017 & 24.00 & 0.0013 & 20.44 & 0.0012 & 16.49 & 0.0017 & 24.65 & 0.0013 & 26.12 \\
\hline 0.0019 & 27.00 & 0.0015 & 24.37 & 0.0014 & 19.98 & 0.0020 & 28.36 & 0.0017 & 31.07 \\
\hline 0.0020 & 28.00 & 0.0018 & 27.71 & 0.0016 & 22.89 & 0.0022 & 32.07 & 0.0021 & 35.51 \\
\hline 0.0022 & 32.00 & 0.0019 & 31.64 & 0.0019 & 27.16 & 0.0026 & 35.13 & 0.0024 & 37.85 \\
\hline 0.0023 & 34.00 & 0.0023 & 34.19 & 0.0020 & 29.68 & 0.0029 & 38.85 & 0.0026 & 41.50 \\
\hline 0.0023 & 36.00 & 0.0025 & 35.37 & 0.0022 & 34.53 & 0.0033 & 41.04 & 0.0029 & 45.41 \\
\hline 0.0026 & 35.00 & 0.0027 & 37.14 & 0.0025 & 36.27 & 0.0034 & 43.00 & 0.0034 & 47.74 \\
\hline 0.0028 & 33.00 & 0.0029 & 40.28 & 0.0027 & 37.44 & 0.0036 & 44.10 & 0.0036 & 48.51 \\
\hline 0.0031 & 30.00 & 0.0030 & 40.87 & 0.0030 & 39.57 & 0.0037 & 44.97 & 0.0038 & 49.29 \\
\hline 0.0037 & 15.00 & 0.0032 & 40.87 & 0.0032 & 41.51 & 0.0040 & 44.99 & 0.0040 & 51.63 \\
\hline & & 0.0037 & 38.91 & 0.0036 & 41.70 & 0.0044 & 41.75 & 0.0042 & 52.93 \\
\hline & & 0.0040 & 34.78 & 0.0042 & 39.76 & 0.0047 & 39.59 & 0.0045 & 53.17 \\
\hline & & 0.0042 & 30.07 & 0.0046 & 36.27 & 0.0049 & 36.56 & 0.0049 & 49.74 \\
\hline & & 0.0046 & 27.51 & 0.0051 & 33.36 & & & 0.0051 & 45.27 \\
\hline & & 0.0048 & 24.96 & 0.0055 & 30.06 & & & 0.0053 & 40.55 \\
\hline & & & & 0.0057 & 26.96 & & & & \\
\hline
\end{tabular}

Table 5. Stress-strain values of glass fibre reinforced self-compacting concrete (GFRSCC) mixes made with

$\mathrm{PF}=1.14$ and $\mathrm{s} / \mathrm{a}=0.57$ in confined state

\begin{tabular}{|c|c|c|c|c|c|c|c|c|c|}
\hline \multicolumn{10}{|c|}{$\mathrm{PF}=1.14$ and $\mathrm{s} / \mathrm{a}=0.57$} \\
\hline \multicolumn{2}{|c|}{ M30GFRSCC-C0 } & \multicolumn{2}{|c|}{ M30GFRSCC-C3 } & \multicolumn{2}{|c|}{ M30GFRSCC-C4 } & \multicolumn{2}{|c|}{ M30GFRSCC-C5 } & \multicolumn{2}{|c|}{ M30GFRSCC-C6 } \\
\hline Strain & Stress & Strain & Stress & Strain & Stress & Strain & Stress & Strain & Stress \\
\hline 0.0000 & 0.00 & 0.0000 & 0.00 & 0.0000 & 0.00 & 0.0000 & 0.00 & 0.0000 & 0.00 \\
\hline 0.0001 & 3.52 & 0.0000 & 0.43 & 0.0001 & 0.21 & 0.0000 & 0.52 & 0.0000 & 0.00 \\
\hline 0.0003 & 6.46 & 0.0003 & 5.16 & 0.0002 & 3.93 & 0.0002 & 5.98 & 0.0001 & 3.13 \\
\hline 0.0005 & 10.37 & 0.0005 & 10.10 & 0.0005 & 8.51 & 0.0004 & 10.66 & 0.0002 & 6.77 \\
\hline 0.0010 & 16.83 & 0.0007 & 13.54 & 0.0007 & 12.21 & 0.0007 & 15.60 & 0.0005 & 10.68 \\
\hline 0.0012 & 20.74 & 0.0011 & 18.26 & 0.0009 & 17.02 & 0.0010 & 20.54 & 0.0007 & 15.88 \\
\hline 0.0016 & 24.85 & 0.0013 & 21.69 & 0.0013 & 21.36 & 0.0012 & 26.00 & 0.0010 & 20.84 \\
\hline 0.0018 & 28.37 & 0.0015 & 25.99 & 0.0016 & 26.15 & 0.0014 & 30.16 & 0.0012 & 25.52 \\
\hline 0.0021 & 32.48 & 0.0018 & 28.99 & 0.0018 & 28.76 & 0.0017 & 33.53 & 0.0014 & 29.44 \\
\hline 0.0024 & 35.41 & 0.0021 & 32.63 & 0.0021 & 32.89 & 0.0019 & 37.68 & 0.0017 & 33.61 \\
\hline 0.0025 & 35.80 & 0.0024 & 35.85 & 0.0024 & 36.37 & 0.0022 & 40.53 & 0.0019 & 37.00 \\
\hline 0.0028 & 40.11 & 0.0026 & 39.07 & 0.0027 & 39.41 & 0.0026 & 43.90 & 0.0021 & 40.38 \\
\hline 0.0031 & 40.11 & 0.0030 & 41.85 & 0.0030 & 40.70 & 0.0030 & 45.96 & 0.0023 & 43.26 \\
\hline
\end{tabular}




\begin{tabular}{|l|l|l|l|l|l|l|l|l|l|}
\hline 0.0034 & 38.93 & 0.0032 & 43.34 & 0.0032 & 42.87 & 0.0033 & 46.98 & 0.0027 & 46.65 \\
\hline 0.0038 & 35.41 & 0.0035 & 42.25 & 0.0036 & 45.68 & 0.0034 & 47.75 & 0.0028 & 48.22 \\
\hline 0.0040 & 31.89 & 0.0038 & 39.21 & 0.0039 & 44.77 & 0.0037 & 45.90 & 0.0032 & 49.80 \\
\hline 0.0042 & 28.76 & 0.0039 & 36.40 & 0.0042 & 40.57 & 0.0040 & 44.31 & 0.0038 & 52.43 \\
\hline 0.0044 & 25.83 & 0.0041 & 34.24 & 0.0045 & 35.50 & 0.0043 & 40.90 & 0.0041 & 53.49 \\
\hline 0.0045 & 24.07 & 0.0044 & 32.07 & 0.0046 & 33.08 & 0.0045 & 38.80 & 0.0043 & 54.28 \\
\hline & & 0.0045 & 30.55 & 0.0047 & 28.68 & 0.0046 & 36.45 & 0.0044 & 53.51 \\
\hline & & & & 0.0048 & 25.81 & & & 0.0046 & 52.49 \\
\hline & & & & & & & & 0.0049 & 50.94 \\
\hline & & & & & & & & 0.0050 & 48.61 \\
\hline
\end{tabular}

Table 6. Stress-strain values of steel fibre reinforced self-compacting concrete ( $\mathrm{SFRSCC}$ ) mixes made with $\mathrm{PF}=1.12$ and s/a=0.53 in confined state

\begin{tabular}{|c|c|c|c|c|c|c|c|c|c|}
\hline \multicolumn{10}{|c|}{ PF=1.12 and s/a $=0.53$} \\
\hline M30SFRSCC-C0 & \multicolumn{1}{|c|}{ M30SFRSCC-C3 } & \multicolumn{2}{|c|}{ M30SFRSCC-C4 } & \multicolumn{2}{l|}{ M30SFRSCC-C } & \multicolumn{2}{c|}{ M30SFRSCC-C6 } \\
\hline Strain & Stress & Strain & Stress & Strain & Stress & Strain & Stress & Strain & Stress \\
\hline 0.0000 & 0.00 & 0.0000 & 0.00 & 0.0000 & 0.00 & 0.0000 & 0.00 & 0.0000 & 0.00 \\
\hline 0.0003 & 6.43 & 0.0003 & 5.05 & 0.0002 & 4.77 & 0.0003 & 5.41 & 0.0003 & 6.75 \\
\hline 0.0005 & 10.91 & 0.0006 & 10.11 & 0.0005 & 9.54 & 0.0004 & 9.74 & 0.0006 & 10.91 \\
\hline 0.0008 & 15.19 & 0.0009 & 13.21 & 0.0007 & 14.53 & 0.0008 & 16.45 & 0.0010 & 16.62 \\
\hline 0.0011 & 19.09 & 0.0010 & 16.12 & 0.0009 & 19.09 & 0.0011 & 21.00 & 0.0012 & 21.04 \\
\hline 0.0016 & 24.35 & 0.0012 & 19.23 & 0.0012 & 23.42 & 0.0014 & 26.41 & 0.0017 & 26.49 \\
\hline 0.0018 & 27.66 & 0.0015 & 22.92 & 0.0014 & 27.97 & 0.0017 & 30.74 & 0.0021 & 34.81 \\
\hline 0.0020 & 30.00 & 0.0018 & 27.19 & 0.0016 & 31.44 & 0.0018 & 33.55 & 0.0025 & 39.48 \\
\hline 0.0023 & 33.51 & 0.0021 & 30.49 & 0.0020 & 35.55 & 0.0020 & 36.15 & 0.0029 & 43.38 \\
\hline 0.0025 & 36.62 & 0.0022 & 34.58 & 0.0023 & 38.58 & 0.0024 & 38.96 & 0.0032 & 47.27 \\
\hline 0.0027 & 38.96 & 0.0024 & 37.88 & 0.0027 & 41.17 & 0.0026 & 41.99 & 0.0037 & 51.95 \\
\hline 0.0031 & 38.57 & 0.0027 & 41.56 & 0.0030 & 41.58 & 0.0030 & 42.86 & 0.0040 & 53.51 \\
\hline 0.0035 & 36.43 & 0.0028 & 41.75 & 0.0032 & 42.66 & 0.0033 & 43.51 & 0.0043 & 54.55 \\
\hline 0.0038 & 32.73 & 0.0032 & 42.32 & 0.0034 & 43.74 & 0.0035 & 45.67 & 0.0044 & 55.84 \\
\hline 0.0041 & 29.42 & 0.0037 & 41.33 & 0.0037 & 41.77 & 0.0039 & 45.24 & 0.0048 & 55.32 \\
\hline 0.0043 & 28.64 & 0.0040 & 38.78 & 0.0040 & 36.54 & 0.0042 & 42.21 & 0.0053 & 51.17 \\
\hline 0.0045 & 24.94 & 0.0044 & 34.86 & 0.0041 & 33.27 & 0.0045 & 40.48 & 0.0056 & 48.31 \\
\hline 0.0046 & 22.60 & 0.0046 & 31.55 & 0.0045 & 30.64 & 0.0048 & 38.74 & 0.0059 & 45.19 \\
\hline 0.0048 & 19.68 & 0.0047 & 28.42 & 0.0046 & 27.37 & 0.0049 & 37.01 & & \\
\hline & & 0.0048 & 26.28 & & & & & & \\
\hline
\end{tabular}

Table 7. Stress-strain values of steel fibre reinforced self-compacting concrete (SFRSCC) mixes made with $\mathrm{PF}=1.14$ and s/a=0.57 in confined state

\begin{tabular}{|c|c|c|c|c|c|c|c|c|c|}
\hline \multicolumn{10}{|c|}{$\mathrm{PF}=1.14$ and $\mathrm{s} / \mathrm{a}=0.57$} \\
\hline \multicolumn{2}{|c|}{ M30SFRSCC-C0 } & \multicolumn{2}{|c|}{ M30SFRSCC-C0 } & \multicolumn{2}{|c|}{ M30SFRSCC-C0 } & \multicolumn{2}{|c|}{ M30SFRSCC-C0 } & \multicolumn{2}{|c|}{ M30SFRSCC-C0 } \\
\hline Strain & Stress & Strain & Stress & Strain & Stress & Strain & Stress & Strain & Stress \\
\hline 0.0000 & 0.00 & 0.0000 & 0.00 & 0.0000 & 0.00 & 0.0000 & 0.00 & 0.0000 & 0.00 \\
\hline 0.0000 & 0.65 & 0.0003 & 6.26 & 0.0000 & 0.26 & 0.0000 & 0.52 & 0.0001 & 0.26 \\
\hline 0.0002 & 4.33 & 0.0005 & 11.01 & 0.0002 & 4.69 & 0.0001 & 4.19 & 0.0002 & 6.29 \\
\hline 0.0004 & 8.44 & 0.0007 & 14.03 & 0.0003 & 8.85 & 0.0003 & 8.65 & 0.0005 & 11.00 \\
\hline 0.0006 & 11.47 & 0.0009 & 17.26 & 0.0007 & 14.83 & 0.0005 & 12.31 & 0.0007 & 13.89 \\
\hline 0.0007 & 14.07 & 0.0012 & 22.01 & 0.0009 & 19.51 & 0.0008 & 16.77 & 0.0010 & 18.86 \\
\hline 0.0010 & 16.67 & 0.0016 & 27.83 & 0.0012 & 23.41 & 0.0009 & 21.22 & 0.0012 & 22.53 \\
\hline 0.0012 & 19.05 & 0.0019 & 34.08 & 0.0015 & 27.30 & 0.0012 & 24.37 & 0.0016 & 29.08 \\
\hline 0.0013 & 21.65 & 0.0022 & 37.53 & 0.0017 & 31.73 & 0.0014 & 29.34 & 0.0021 & 37.21 \\
\hline 0.0016 & 25.32 & 0.0026 & 40.75 & 0.0019 & 34.58 & 0.0017 & 32.75 & 0.0024 & 41.66 \\
\hline
\end{tabular}




\begin{tabular}{|l|l|l|l|l|l|l|l|l|l|}
\hline 0.0019 & 29.87 & 0.0030 & 43.11 & 0.0022 & 37.95 & 0.0018 & 36.16 & 0.0028 & 45.07 \\
\hline 0.0022 & 32.90 & 0.0033 & 46.12 & 0.0025 & 41.59 & 0.0022 & 39.56 & 0.0032 & 48.73 \\
\hline 0.0024 & 35.28 & 0.0036 & 45.67 & 0.0028 & 43.14 & 0.0023 & 42.97 & 0.0035 & 50.83 \\
\hline 0.0027 & 38.10 & 0.0038 & 43.93 & 0.0030 & 45.47 & 0.0027 & 45.59 & 0.0040 & 52.40 \\
\hline 0.0030 & 41.99 & 0.0041 & 41.31 & 0.0032 & 47.80 & 0.0030 & 47.42 & 0.0042 & 52.66 \\
\hline 0.0031 & 42.86 & 0.0042 & 39.14 & 0.0035 & 49.09 & 0.0033 & 48.47 & 0.0046 & 53.97 \\
\hline 0.0034 & 40.04 & 0.0045 & 35.45 & 0.0041 & 49.58 & 0.0035 & 51.09 & 0.0049 & 53.45 \\
\hline 0.0036 & 34.63 & & & 0.0045 & 47.72 & 0.0037 & 51.62 & 0.0054 & 50.83 \\
\hline 0.0040 & 29.00 & & & 0.0048 & 44.58 & 0.0039 & 52.40 & 0.0059 & 45.59 \\
\hline 0.0043 & 22.08 & & & 0.0050 & 41.17 & 0.0043 & 50.83 & & \\
\hline 0.0044 & 16.67 & & & & & 0.0046 & 47.16 & & \\
\hline 0.0045 & 12.55 & & & & & 0.0049 & 45.07 & & \\
\hline & & & & & & 0.0052 & 42.97 & & \\
\hline
\end{tabular}

Table 8. Stress-strain values of hybrid fibre reinforced self-compacting concrete ( $\mathrm{SFRSCC}$ ) mixes made with $\mathrm{PF}=1.12$ and $\mathrm{s} / \mathrm{a}=0.53 \mathrm{in}$ confined state

\begin{tabular}{|c|c|c|c|c|c|c|c|c|c|}
\hline \multicolumn{10}{|c|}{$\mathrm{PF}=1.12$ and $\mathrm{s} / \mathrm{a}=0.53$} \\
\hline \multicolumn{2}{|c|}{ M30HFRSCC-C0 } & \multicolumn{2}{|c|}{ M30HFRSCC-C3 } & \multicolumn{2}{|c|}{ M30HFRSCC-C4 } & \multicolumn{2}{|c|}{ M30HFRSCC-C5 } & \multicolumn{2}{|c|}{ M30HFRSCC-C6 } \\
\hline Strain & Stress & Strain & Stress & Strain & Stress & Strain & Stress & Strain & Stress \\
\hline 0.0000 & 0.00 & 0.0000 & 0.00 & 0.0000 & 0.00 & 0.0000 & 0.00 & 0.0000 & 0.00 \\
\hline 0.0000 & 0.58 & 0.0000 & 0.22 & 0.0001 & 0.26 & 0.0001 & 0.78 & 0.0001 & 0.26 \\
\hline 0.0002 & 4.08 & 0.0002 & 3.04 & 0.0003 & 6.98 & 0.0002 & 7.30 & 0.0003 & 4.92 \\
\hline 0.0003 & 9.34 & 0.0004 & 7.81 & 0.0005 & 12.67 & 0.0005 & 12.00 & 0.0006 & 11.66 \\
\hline 0.0004 & 12.84 & 0.0005 & 12.15 & 0.0007 & 17.59 & 0.0008 & 18.26 & 0.0010 & 19.95 \\
\hline 0.0007 & 17.11 & 0.0006 & 16.92 & 0.0011 & 24.31 & 0.0011 & 23.74 & 0.0014 & 27.72 \\
\hline 0.0010 & 19.83 & 0.0008 & 20.82 & 0.0014 & 29.22 & 0.0014 & 26.35 & 0.0017 & 32.90 \\
\hline 0.0012 & 22.93 & 0.0010 & 26.03 & 0.0018 & 33.62 & 0.0016 & 30.26 & 0.0020 & 37.56 \\
\hline 0.0013 & 25.07 & 0.0012 & 29.50 & 0.0022 & 37.76 & 0.0019 & 34.96 & 0.0025 & 42.73 \\
\hline 0.0015 & 27.20 & 0.0015 & 32.76 & 0.0025 & 41.38 & 0.0023 & 39.13 & 0.0028 & 47.13 \\
\hline 0.0017 & 29.73 & 0.0017 & 35.58 & 0.0027 & 44.48 & 0.0025 & 41.48 & 0.0031 & 50.75 \\
\hline 0.0019 & 31.86 & 0.0020 & 37.98 & 0.0030 & 45.52 & 0.0028 & 43.83 & 0.0034 & 52.81 \\
\hline 0.0021 & 33.80 & 0.0022 & 41.45 & 0.0033 & 47.07 & 0.0031 & 45.91 & 0.0039 & 54.61 \\
\hline 0.0024 & 35.54 & 0.0025 & 42.33 & 0.0034 & 48.88 & 0.0033 & 48.00 & 0.0040 & 54.60 \\
\hline 0.0027 & 39.03 & 0.0027 & 43.43 & 0.0037 & 47.59 & 0.0038 & 48.78 & 0.0043 & 54.84 \\
\hline 0.0030 & 41.16 & 0.0030 & 44.31 & 0.0040 & 43.45 & 0.0042 & 46.43 & 0.0045 & 56.39 \\
\hline 0.0033 & 39.01 & 0.0032 & 45.83 & 0.0045 & 40.86 & 0.0045 & 44.61 & 0.0049 & 56.37 \\
\hline 0.0035 & 35.30 & 0.0035 & 46.93 & 0.0048 & 39.31 & 0.0047 & 43.30 & 0.0053 & 52.71 \\
\hline 0.0036 & 32.17 & 0.0039 & 45.65 & 0.0051 & 37.50 & 0.0049 & 41.74 & 0.0056 & 49.32 \\
\hline 0.0038 & 30.02 & 0.0041 & 44.14 & & & & & 0.0059 & 47.49 \\
\hline 0.0039 & 26.12 & 0.0043 & 41.13 & & & & & & \\
\hline 0.0041 & 22.22 & 0.0046 & 37.03 & & & & & & \\
\hline & & 0.0048 & 34.22 & & & & & & \\
\hline
\end{tabular}

Table 8. Stress-strain values of hybrid fibre reinforced self-compacting concrete (SFRSCC) mixes made with

$\mathrm{PF}=1.14$ and $\mathrm{s} / \mathrm{a}=0.57$ in confined state

\begin{tabular}{|c|c|c|c|c|c|c|c|c|c|}
\hline \multicolumn{10}{|c|}{$\mathrm{PF}=1.14$ and s/a=0.57 } \\
\hline M30HFRSCC-C0 & M30HFRSCC-C3 & \multicolumn{2}{|c|}{ M30HFRSCC-C4 } & M30HFRSCC-C5 & \multicolumn{2}{c|}{ M30HFRSCC-C6 } \\
\hline Strain & Stress & Strain & Stress & Strain & Stress & Strain & Stress & Strain & Stress \\
\hline 0.0000 & 0.00 & 0.0000 & 0.00 & 0.0000 & 0.00 & 0.0000 & 0.00 & 0.0000 & 0.00 \\
\hline 0.0002 & 3.88 & 0.0003 & 4.61 & 0.0000 & 0.78 & 0.0002 & 4.96 & 0.0001 & 0.26 \\
\hline 0.0005 & 9.28 & 0.0005 & 9.21 & 0.0002 & 4.66 & 0.0005 & 11.48 & 0.0002 & 5.23 \\
\hline 0.0007 & 13.59 & 0.0007 & 15.35 & 0.0004 & 10.89 & 0.0007 & 17.22 & 0.0004 & 9.94 \\
\hline 0.0009 & 18.13 & 0.0009 & 19.96 & 0.0006 & 16.59 & 0.0009 & 22.70 & 0.0007 & 14.64 \\
\hline 0.0011 & 22.01 & 0.0012 & 23.68 & 0.0009 & 21.50 & 0.0012 & 28.17 & 0.0010 & 19.08 \\
\hline
\end{tabular}




\begin{tabular}{|c|c|c|c|c|c|c|c|c|c|}
\hline 0.0014 & 25.02 & 0.0014 & 28.07 & 0.0012 & 26.16 & 0.0014 & 33.13 & 0.0012 & 22.99 \\
\hline 0.0016 & 28.48 & 0.0017 & 32.46 & 0.0015 & 30.56 & 0.0017 & 36.52 & 0.0015 & 27.69 \\
\hline 0.0019 & 32.57 & 0.0020 & 35.75 & 0.0017 & 35.22 & 0.0019 & 39.91 & 0.0017 & 32.40 \\
\hline 0.0021 & 35.59 & 0.0023 & 37.72 & 0.0019 & 38.84 & 0.0021 & 42.52 & 0.0019 & 35.79 \\
\hline 0.0024 & 37.95 & 0.0025 & 39.91 & 0.0023 & 41.42 & 0.0024 & 45.13 & 0.0023 & 39.18 \\
\hline 0.0027 & 40.96 & 0.0027 & 42.54 & 0.0027 & 44.51 & 0.0027 & 48.00 & 0.0024 & 41.79 \\
\hline 0.0030 & 43.54 & 0.0030 & 44.96 & 0.0030 & 47.61 & 0.0030 & 50.09 & 0.0026 & 46.24 \\
\hline 0.0030 & 44.19 & 0.0032 & 46.27 & 0.0032 & 48.38 & 0.0032 & 50.87 & 0.0029 & 48.06 \\
\hline 0.0033 & 43.74 & 0.0034 & 46.93 & 0.0036 & 50.43 & 0.0035 & 52.43 & 0.0033 & 50.40 \\
\hline 0.0036 & 42.43 & 0.0038 & 45.83 & 0.0038 & 49.64 & 0.0037 & 54.00 & 0.0036 & 52.48 \\
\hline 0.0039 & 39.16 & 0.0042 & 42.32 & 0.0040 & 48.85 & 0.0041 & 54.26 & 0.0039 & 54.82 \\
\hline 0.0042 & 35.03 & 0.0044 & 38.82 & 0.0042 & 47.28 & 0.0043 & 52.70 & 0.0042 & 55.85 \\
\hline 0.0043 & 32.42 & 0.0045 & 36.84 & 0.0046 & 43.88 & 0.0045 & 49.83 & 0.0045 & 55.31 \\
\hline \multirow[t]{5}{*}{0.0045} & 30.68 & & & 0.0047 & 41.79 & 0.0047 & 48.26 & 0.0048 & 56.60 \\
\hline & & & & 0.0049 & 39.70 & 0.0049 & 46.17 & 0.0053 & 54.75 \\
\hline & & & & & & 0.0050 & 44.09 & 0.0055 & 53.42 \\
\hline & & & & & & & & 0.0057 & 51.06 \\
\hline & & & & & & & & 0.0059 & 48.68 \\
\hline
\end{tabular}

Table 9. Peak stress and strain at peak stress values of fibre reinforced self-compacting concrete mixes made with $\mathrm{PF}=1.12$ and $\mathrm{s} / \mathrm{a}=0.53$ in unconfined and confined state

\begin{tabular}{|c|c|c|c|}
\hline Type & Name & $\begin{array}{l}\text { Peak stress } \\
(\mathrm{MPa})\end{array}$ & Strain at peak stress \\
\hline \multirow{5}{*}{$\begin{array}{c}\mathrm{PF}=1.12 \\
\text { and } \\
\mathrm{s} / \mathrm{a}=0.53\end{array}$} & M30PSCC-C0 & 38.31 & 0.0026 \\
\hline & M30PSCC-C3 & 42.48 & 0.0030 \\
\hline & M30PSCC-C4 & 44.91 & 0.0033 \\
\hline & M30PSCC-C5 & 47.06 & 0.0037 \\
\hline & M30PSCC-C6 & 56.76 & 0.0044 \\
\hline \multirow{5}{*}{$\begin{array}{c}\mathrm{PF}=1.12 \\
\text { and } \\
\mathrm{s} / \mathrm{a}=0.53\end{array}$} & M30GFRSCC-C0 & 38.87 & 0.0024 \\
\hline & M30GFRSCC-C3 & 44.48 & 0.0032 \\
\hline & M30GFRSCC-C4 & 45.76 & 0.0034 \\
\hline & M30GFRSCC-C5 & 48.88 & 0.0039 \\
\hline & M30GFRSCC-C6 & 58.26 & 0.0046 \\
\hline \multirow{5}{*}{$\begin{array}{c}\mathrm{PF}=1.12 \\
\text { and } \\
\mathrm{s} / \mathrm{a}=0.53\end{array}$} & M30SFRSCC-C0 & 43.40 & 0.0028 \\
\hline & M30SFRSCC-C3 & 46.46 & 0.0030 \\
\hline & M30SFRSCC-C4 & 47.80 & 0.0036 \\
\hline & M30SFRSCC-C5 & 50.05 & 0.0038 \\
\hline & M30SFRSCC-C6 & 60.63 & 0.0047 \\
\hline \multirow{5}{*}{$\begin{array}{c}\mathrm{PF}=1.12 \\
\text { and } \\
\mathrm{s} / \mathrm{a}=0.53\end{array}$} & M30HFRSCC-C0 & 45.35 & 0.0032 \\
\hline & M30HFRSCC-C3 & 50.18 & 0.0034 \\
\hline & M30HFRSCC-C4 & 52.68 & 0.0036 \\
\hline & M30HFRSCC-C5 & 52.86 & 0.0040 \\
\hline & M30HFRSCC-C6 & 62.33 & 0.0050 \\
\hline
\end{tabular}


Table 9. Peak stress and strain at peak stress values of fibre reinforced self-compacting concrete mixes made with $\mathrm{PF}=1.14$ and $\mathrm{s} / \mathrm{a}=0.57$ in unconfined and confined state

\begin{tabular}{|c|c|c|c|}
\hline Type & Name & $\begin{array}{l}\text { Peak stress } \\
\quad(\mathrm{MPa})\end{array}$ & Strain at peak stress \\
\hline \multirow{5}{*}{$\begin{array}{c}\mathrm{PF}=1.14 \\
\text { and } \\
\mathrm{s} / \mathrm{a}=0.57\end{array}$} & M30PSCC-C0 & 40.93 & 0.0031 \\
\hline & M30PSCC-C3 & 45.84 & 0.0032 \\
\hline & M30PSCC-C4 & 48.42 & 0.0035 \\
\hline & M30PSCC-C5 & 52.61 & 0.0039 \\
\hline & M30PSCC-C6 & 56.65 & 0.0042 \\
\hline \multirow{5}{*}{$\begin{array}{c}\mathrm{PF}=1.14 \\
\text { and } \\
\mathrm{s} / \mathrm{a}=0.57\end{array}$} & M30GFRSCC-C0 & 44.13 & 0.0030 \\
\hline & M30GFRSCC-C3 & 47.52 & 0.0033 \\
\hline & M30GFRSCC-C4 & 49.98 & 0.0038 \\
\hline & M30GFRSCC-C5 & 52.38 & 0.0036 \\
\hline & M30GFRSCC-C6 & 59.13 & 0.0044 \\
\hline \multirow{5}{*}{$\begin{array}{c}\mathrm{PF}=1.14 \\
\text { and } \\
\mathrm{s} / \mathrm{a}=0.57\end{array}$} & M30SFRSCC-C0 & 46.57 & 0.0033 \\
\hline & M30SFRSCC-C3 & 50.29 & 0.0035 \\
\hline & M30SFRSCC-C4 & 53.77 & 0.0037 \\
\hline & M30SFRSCC-C5 & 56.94 & 0.0041 \\
\hline & M30SFRSCC-C6 & 59.33 & 0.0048 \\
\hline \multirow{5}{*}{$\begin{array}{c}\mathrm{PF}=1.14 \\
\text { and } \\
\mathrm{s} / \mathrm{a}=0.57\end{array}$} & M30HFRSCC-C0 & 48.20 & 0.0033 \\
\hline & M30HFRSCC-C3 & 50.37 & 0.0034 \\
\hline & M30HFRSCC-C4 & 55.24 & 0.0038 \\
\hline & M30HFRSCC-C5 & 58.45 & 0.0039 \\
\hline & M30HFRSCC-C6 & 61.56 & 0.0050 \\
\hline
\end{tabular}

\section{Conclusions}

1. The addition of fibres along with confinement of FRSCC with steel hoops enhanced the compressive strength, indicating further confinement effect in the FRSCC.

2. It is observed that the addition of fibres is helpful in lower confinements only. Beyond $1.1 \%$ confinement, the addition of any type of fibres doesn't show any effect on compressive strengths.

3. From the stress-strain behaviour of all types of FRSCC, it is concluded that the ultimate loadcarrying capacity and strains at peak stresses are more in SFRSCC and HFRSCC for mixes up to $1.1 \%$ confinement.

4. The addition of fibres to SCC has increased the ductility in both confined and unconfined states.

\section{References}

1. T.Srinivas and M. Abinay Raj, Int. J. of Eng.and Adv. Tech. (IJEAT), ISSN: 2249 - 8958, Volume-8 Issue-6 (2019)

2. T.srinivas and P. Manoj Anand, Int. J. of Innov. Tech. and Explor. Eng.g (IJITEE), ISSN: 2278-3075, Volume-8 Issue-12 (2019)

3. T.Srinivas and G. Sukesh Reddy, Int. J. of Eng.and Adv. Tech. (IJEAT), ISSN: 2249 - 8958, Volume-9 Issue-1 (2019)
4. T.Srinivas and R. N. Koushik, Int. J. of Innov. Tech. and Explor. Eng.g (IJITEE), ISSN: 2278-3075, Volume-8 Issue-12 (2019), PP 112-117.

5. K. Sai Gopi, Dr. T. Srinivas and S. P. Raju V, E3S Web of Conferences ICMED 184, 01084GRIET, 2829

February, https://doi.org/10.1051/e3sconf/2020184011084(20 20)

6. Jagannadha Kumar, M.V., Jagannadha Rao, K., Dean Kumar, B., Srinivasa Reddy, V., Int. J. of Civil Eng. and Tech., 9(7), pp. 1133-1141 (2018)

7. B. J. Varghese and P. B. Bobba, "2016 IEEE 1st International Conference on Power Electronics, Intelligent Control and Energy Systems (ICPEICES), 2016, pp. 1-5

8. Ganta, J.K., Seshagiri Rao, M.V., Mousavi, S.S., Srinivasa Reddy, V., Bhojaraju, C., Structures 28, pp. 956-972 (2020)

9. Naidu, K.S.S.T., Rao, M.V.S., Reddy, V.S., Int. J. of Innov. Tech. and Explor. Eng.g (IJITEE), 8(9 Special Issue 2), pp. 641-642 (2019)

10. Chandana Priya, C., Seshagiri Rao, M.V., Srinivasa Reddy, V., Int. J. of Civil Eng. and Tech., 9(11), pp. 2218-2225 (2018)

11. Satya Sai Trimurty Naidu, K., Seshagiri Rao, M.V., Srinivasa Reddy, V., Int. J. of Civil Eng. and Tech., 9(11), pp. 2383-2393 (2018)

12. Kavitha, Merugu; Prasad, Dinkar; Bobba, Phaneendra Babu: IET Electric Power Applications, 2019, 13, (8), p. 1184-1192 
13. Supriya, Y., Srinivasa Reddy, V., Seshagiri Rao, M.V., Shrihari, S., Int. J. of Rec. Tech. and Engi., 8(3), pp. 5381-5385 (2019)

14. Kotkunde, N., Krishna, G., Shenoy, S.K., Gupta, A.K., Singh, S.K. International Journal of Material Forming, 10 (2), pp. 255-266 (2017)

15. Govardhan, D., Kumar, A.C.S., Murti, K.G.K., Madhusudhan Reddy, G. Materials and Design, 36, pp. 206-214. (2012)

16. D. Baloji, K. Anil, K. Satyanarayana, S. K. Singh, and M.T. Naik, Materials Today: Proceedings, 18, 4475 (2019).

17. Kumar, P., Singhal, A., Mehta, S., Mittal, A. Journal of Real-Time Image Processing, 11 (1), pp. 93-109. (2016)

18. Srinivas Rao J, S K Tummala, Kuthuri N R, Indonesia Journal of Electrical Engg. \& Computer Science, 21 (723), 2020

19. Raghunadha Reddy, T., Vishnu Vardhan, B., Vijayapal Reddy, P. International Journal of Applied Engineering Research, 11 (5), pp. 3092-3102 (2016)

20. Hussaini, S.M., Krishna, G., Gupta, A.K., Singh, S.K. Journal of Manufacturing Processes, 18, pp. 151-158 (2015) 